\title{
Diagnosis and treatment
}

\author{
COVID-19 Prevention and Control
}




\section{Diagnosis and treatment}

\section{How to detect 2019-nCoV?}

The samples of nasopharyngeal swabs, sputum, lower respiratory tract secretions, blood and feces are collected by medical staff, and real-time fluorescent RT-PCR detects the RNA of 2019-nCoV. Gene sequencing analysis can also be used for diagnosis by comparing with the gene sequence of known 2019-nCoV to see if they are highly homologous. In order to increase the positive rate of nucleic acid detection, it is suggested that sputum should be retained as much as possible, and that lower respiratory secretions should be collected from patients with tracheal intubation. Samples should be submitted for examination as soon as possible after collection.

(Reference: Diagnosis and Treatment Protocol for COVID-19 (Trial Version 6) issued by National Health Commission and National Administration of Traditional Chinese Medicine)

\section{How to diagnose 2019-nCoV infection?}

Suspected cases: a comprehensive analysis is conducted based on epidemiological history and clinical manifestations. Cases that meet any one condition of the epidemiological histories and have any two conditions of the clinical manifestations. If there is no clear history of epidemiology, then the case should meet three conditions of the clinical manifestations.

\section{History of Epidemiology:}

1) Travel or residence history in Wuhan and its surrounding areas or other communities with reported cases within 14 days before the onset of the disease. 
2) History of contact with 2019-nCoV infected patients (the result of nucleic acid test is positive) within 14 days before the onset of the disease.

3) Contact with patients with fever or respiratory symptoms from Wuhan and its surrounding areas or other communities with reported cases within 14 days before the onset of the disease.

4) Cluster infection.

\section{Clinical manifestations:}

1) Fever and / or respiratory symptoms;

2) Having the imaging features of COVID-19: multiple patchy shadows and interstitial changes are found in the early stage, especially in the lung periphery. And then the conditions develop into multiple ground-glass opacifications (GGO) and infiltration shadows. Severe cases may develop consolidation of lung tissue, with incidence of hydrothorax being rare;

3) The total leukocyte count is normal or decreases, or the lymphocyte count decreases in the early stage of the disease.

\section{Confirmed case: suspected case with one of the following etiological evidences:}

1) The result of RT-PCR used to detect the nucleic acid of 2019-nCoV is positive;

2) The result of virus gene sequencing analysis is highly homologous with the known 2019-nCoV.

(Reference: Diagnosis and Treatment Protocol for COVID-19 (Trial Version 6) issued by National Health Commission and National Administration of Traditional Chinese Medicine) 


\section{Symptoms after 2019-nCoV infection, what attention should be paid to when 2019-nCoV infected patients go to the hospital?}

After being infected with the 2019-nCoV, mild cases only show low-grade fever, mild fatigue, and no signs of pneumonia. The main clinical manifestations of 2019-nCoV infection are fever, fatigue, and dry cough. A few patients have symptoms such as nasal congestion, runny nose, sore throat, myalgia and diarrhea. In many severe patients, breathing difficulty and/or hypoxemia occurs after one week, and those critical cases can quickly progress to acute respiratory distress syndrome, septic shock, and metabolic acidosis and coagulation dysfunction that are difficult to be corrected, and multiple organ dysfunction syndrome (MODS), etc. Notably, severe and critical patients may have moderate to lowgrade fever or even no obvious fever during the course of the disease.

If suspected of COVID-19, the hospitals will transfer the patients to the designated hospital for quarantine treatment, and carry out etiological detection. The patient should provide a truthful and detailed account of the disease situation and the history of seeking medical treatment, especially recent history of travel and residence in Wuhan, history of contact with patients with infected or suspected patients, history of animal contact, and so on.

(Reference: Diagnosis and Treatment Protocol for COVID-19 (Trial Version 6) issued by National Health Commission and National Administration of Traditional Chinese Medicine; PUMCH COVID-19 Prevention and Precautions Handbook issued by Chinese Academy of Medical Sciences and Peking Union Medical College Hospital)

\section{What are the treatments for COVID-19?}

Suspected and confirmed cases should be isolated and treated in designated hospitals with effective isolation and protective conditions, and suspected cases should be isolated in a single room. Multiple confirmed cases can be admitted to the same ward, and critical cases should be admitted to ICU as soon as possible. 
General treatments: patients should rest on the bed and receive strengthened supportive care to ensure sufficient energy intake; attention should be paid to water and electrolyte balance to maintain the stability

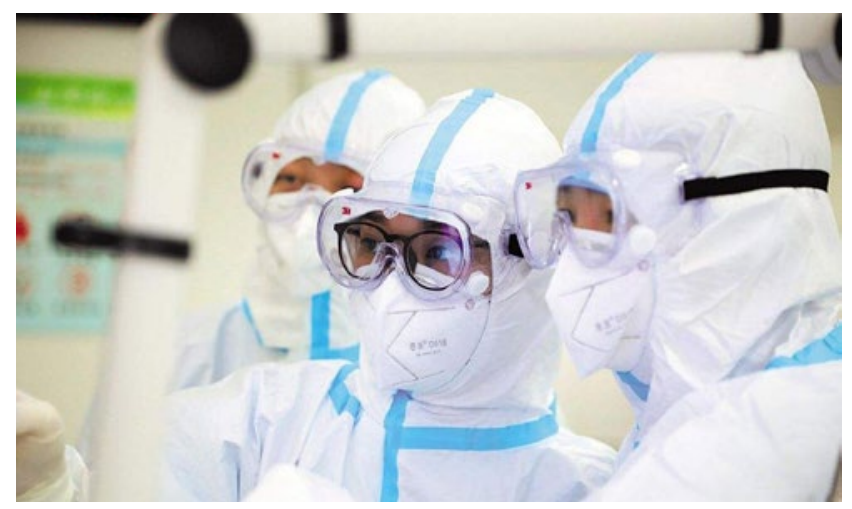
of the internal environment, and vital signs and finger oxygen saturation should be closely monitored.

Complete blood count, urinalysis, CRP, biochemical indicators (liver enzyme, myocardial enzyme, renal function, etc.), blood coagulation function, arterial blood gas analysis, chest imaging should be monitored according to the conditions of the cases. Cytokines testing should be performed if conditions permit.

Effective oxygen therapy measures should be provided in time, including nasal cannula, oxygen masks and high-flow nasal cannula oxygen therapy.

Anti-virus treatment: doctors may administer atomization inhalation of Interferon- $\alpha$ (for adults: 5 million $U$ or equivalent dose, added with $2 \mathrm{ml}$ sterile injection water, atomization inhalation twice a day), Lopinavir/ Ritonavir (for adults: $200 \mathrm{mg} / 50 \mathrm{mg}$ per pill, 2 pills per serving, twice a day, the course of treatment should not exceed 10 days), Ribavirin (it is recommended to use it in combination with Interferon- $\alpha$ or Lopinavir/ Ritonavir, for adults $500 \mathrm{mg}$ each injection, iv. two to three times a day, the course of treatment should not exceed 10 days). Chloroquine phosphate (for adults: $500 \mathrm{mg}$, twice a day, the course of treatment should not exceed 10 days) and Arbidol (for adults: $200 \mathrm{mg}$, three times a day, the course of treatment should not exceed 10 days).

Attention should be paid to the adverse reaction associated with Lopinavir/ Ritonavir such 
as diarrhea, nausea, vomiting, and liver function impairment and also the interaction with other drugs. Further evaluate the efficacy of these trial drugs in clinical applications. It is not recommended to use 3 or more antiviral drugs at the same time, and drugs should be stopped when intolerable side effects occur.

Avoid inappropriate use of antibiotics, especially the combination with broad-spectrum antibiotics.

(Reference: Diagnosis and Treatment Protocol for COVID-19 (Trial Version 6) issued by National Health Commission and National Administration of Traditional Chinese Medicine)

\section{What is the role of traditional Chinese medicine} (TMC) in treating patients with COVID-19?

This disease belongs to the category of plague in traditional Chinese medicine (TCM), caused by the epidemic pathogenic factors. According to the recent clinical treatment and efficacy observations of traditional Chinese medicine, on February 6, 2020, the General Office of the National Health Committee and the Office of

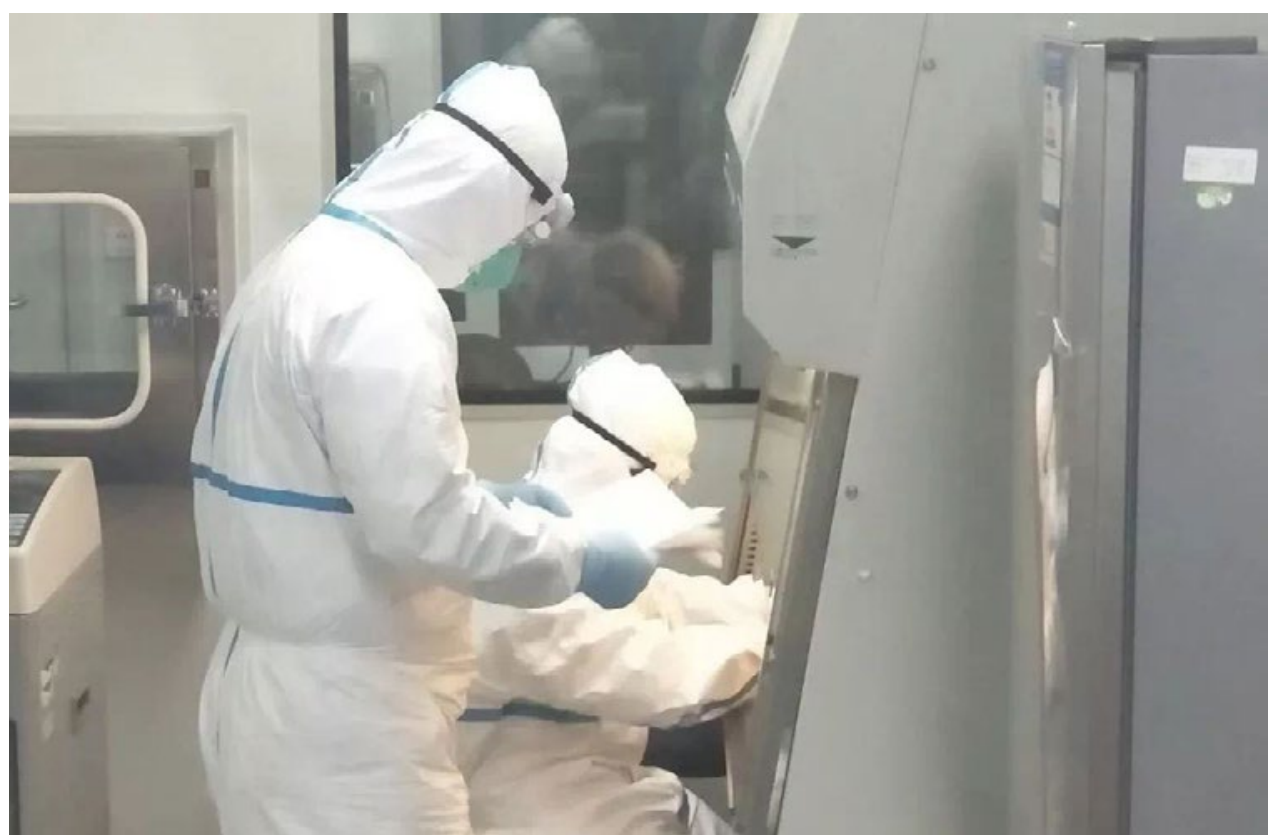


the State Administration of Traditional Chinese Medicine recommended "Lung cleansing \& detoxifying decoction" for use in all regions.

Recommended prescription: Ephedra 9g, Zhigancao 6g, Almond 9g, Gypsum 15-30g (fried first), Guizhi 9g, Zixie 9g, Zhuling 9g, Baizhu 9g, Zhiling 15g, Bupleurum 16g, Scutellaria baicalensis 6g, and Pinellia 9g , Ginger 9g, aster 9g, winter flower $9 \mathrm{~g}$, shoot dry $9 \mathrm{~g}$, asarum $6 \mathrm{~g}$, yam $12 \mathrm{~g}$, coriander fruit $6 \mathrm{~g}$, tangerine peel $6 \mathrm{~g}$, aquilegia $9 \mathrm{~g}$.

Suggested use: Traditional Chinese medicine decoction pieces for decocting in water. One dose per day, twice in the morning and evening (forty minutes after a meal), take with warm water, and three doses a course.

If conditions permit, the patient can take half a bowl of rice soup each time after taking the medicine, and can take up to one bowl if the patient has a dry tongue and is deficient in bodily fluids. (Note: If the patient does not have a fever, the amount of gypsum should be little. If having a fever or strong heat, the amount of gypsum can be increased). If the symptoms improve but do not fully recover, then take the second course of treatment. If the patient has special conditions or other underlying diseases, the prescription of the second course of treatment can be modified based on the actual situation and the medicine should be discontinued when the symptoms disappear.

Scope of application: It is suitable for light, ordinary and severe patients, and can be used reasonably in combination with the actual situation of patients in the treatment of critically ill patients. 


\section{What is the prognosis of infected patients?}

According to the cases currently treated, most of the patients have a good prognosis and a few are in severe condition. The prognosis of the elderly and patients with chronic underlying diseases is relatively weak and the symptoms of children are relatively mild. After discharge, the patient is at risk of infection with other pathogens due to low immune function in convalescence.

(Reference: Diagnosis and Treatment Protocol for COVID-19 (Trial Version 6) issued by National Health Commission and National Administration of Traditional Chinese Medicine)

\section{Are discharged patients still contagious and will they be infected again?}

The designated hospitals should make good contact with the grass-roots medical institutions where the patients live, share the medical records and discharged patient information to the patient's jurisdiction or the residential committee and the grass-roots medical and health institutions on time.

After discharge, the patient is at risk of infection with other pathogens due to low immune function in convalescence. Therefore, it is recommended to continue to monitor self-health for 14 days, wear a mask, and if possible, live in a single room with good ventilation. Avoid close contact with family members, eat separately, keep hands clean and avoid outdoor activities.

It is recommended to go to the hospital for a follow-up visit at the 2nd and 4th week after discharge.

(Reference: Diagnosis and Treatment Protocol for COVID-19 (Trial Version 6) issued by National Health Commission and National Administration of Traditional Chinese Medicine) 